Only after operation was she set on the road to recovery. It is noteworthy that the insomnia, dyspepsia, and the morbid habit of magnifying the noises in her head did not disappear immediately after the operation but improved over a number of months concurrently with the remarkable change in her appearance. It seems reasonable to suppose that the rejuvenating effect of the operation enabled the patient gradually to emerge from her hypochondriacal state.

\section{TREATMENT OF IMPETIGO}

\section{THE VIRTUES OF CALAMINE LINIMFNT AND SOME MINOR DRAWBACKS OF LOCAL SULPHONAMIDE THERAPY}

BY

\section{MARY S. SMITH, M.D. \\ Major, R.A.M.C. \\ AND \\ E. COLIN JONES, M.B. \\ Major, R.A.M.C.}

(From a Military Skin Hospital)

No one with experience of treating impetigo can fail to have been impressed at some time or other by the disastrous effects of using a remedy to which the patient's skin happens to be intolerant. First of all there is the major disaster of sulphonamide sensitization, in which the unlucky patient, after weeks or months of treatment, finds himself sensitized, perhaps incurably, to exposure to ordinary daylight, not to mention half a dozen of the potential irritants of a chemical nature he may have encountered during his illness.

But we are not so much concerned with dangers that are well known as with the accidents of therapy that may conceal their nature under the guise of an intransigent malady that persists in getting worse in spite of our treatment, or that somehow just does not get well. Such events are much more common than may be imagined. It is always well to remember the four things that a given local treatment can do: it can, of course, do nothing at all; it can make the disease better; it can make it worse; and it can prevent it from getting well.

It is the last possibility by which one is so often deceived. We therefore venture to draw attention to a mode of treatment which, incidentally, we claim to give results superior to all others in our own hands, but which also has the great virtue of avoiding the application to the skin of antiseptics or other substances capable of irritating it or giving rise to sensitization. Its main disadvantages are the extravagant use of dressings and the need for considerable skill and care in their application; but these are decisively outweighed by the reduction of the time spent in hospital, and the entire avo:dance of disastrous sensitization phenomena.

\section{The Calamine Liniment Method}

Our method rests on six principles: (1) The free exposure of all parts to treatment-by shaving, if necessary. (2) The avoidance of trauma (apart from that occasioned by shaving). (3) Ensuring that the dressings are kept in close apposition with the affected parts, and that they remain moist. (4) That they are removed only for the few minutes necessary for shaving or re-dressing, and immediately reapplied. (5) That every outlying lesion is included in the treatment and that the dressing extends an inch or so beyond the affected area. (6) Finally, that the method of applying the remedy is altered as occasion demands-e.g., from compresses to smears--not the remedy itself.

Since each of these principles is of importance and neglect to observe any one may result in failure, we feel justified in entering into more detailed explanation of some of them.

Exposure of Affected Parts.-Ruthless shaving is, in our opinion, the key to success. On admission all hairy areas which are crusted are shaved, no matter how raw. To offer a little protection to the tender area, and to aid in softening the crusts, the parts are first anointed with lanette wax base ; a lather of soap and water may or may not be used. No attempt should be made to save the hair of the scalp or eye- brows if involved. They should be thoroughly shaved, as even a small tuft of hair overlooked will delay cure. The eyebrows grow rapidly; and in the presence of persistent crusting it may be necessary to re-shave. Over the bearded region this should be done every second day.

\begin{tabular}{|c|c|c|c|c|c|c|c|}
\hline \multicolumn{8}{|c|}{ Lanette Wax Base } \\
\hline Lanette wax & & .. & .. & $\ldots$ & . & 10 & parts \\
\hline line & . & . & .. & .. & . & 10 & 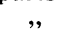 \\
\hline & $\cdots$ & 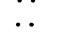 & 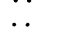 & 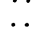 & $\cdots$ & $\ldots$ ad 60 & \\
\hline \multicolumn{8}{|c|}{ Modified Calamine } \\
\hline Adeps lanae & . & $\ldots$ & • & $\cdots$ & . & . . & \\
\hline Lanette wax & .. & . & $\ldots$ & & $\cdots$ & $\cdots$ & \\
\hline Calamine & . & . & . & . & $\cdots$ & . & \\
\hline Olive oil & $\cdots$ & $\cdots$ & . & . & $\cdots$ & . & ,' \\
\hline Water .. & & . & . & & . &. ad 80 & \\
\hline
\end{tabular}

Avoidance of Trauma.-To shave and to avoid trauma are apparently contradictory instructions. The damage caused by shaving is outweighed by the rapid healing which results from the closer contact secured for the dressing. Apart from shaving, no attempt is made to encourage the removal of crusts. Those crusts which do not separate readily and come away with the first dressing are not interfered with, as they seldom fail to do so with subsequent dressings, leaving a surface rapidly healing or healed.

Dressings.-For the dressing to be effective the closest contact is essential. Folds of gauze, four to eight layers thick, are completely saturated with calamine liniment and moulded on to the affected parts. Apposition in the natural folds is secured by packing and firm bandaging. The ear presents special difficulty. Several layers of gauze in the form of a tape are well saturated and tucked into all the natural folds and crannies of the ear. A similar dressing is made to encircle the ear to prevent the fissuring which so readily occurs at the upper and lower poles and in the retro-auricular fold. Unfortunately the ear dressing sometimes causes earache. To obviate this a dry plug of cotton-wool is inserted into the meatus and changed with each dressing. If the skin infection extends into the meatus the plug can be saturated with the liniment. To reach the deeper parts, otherwise inaccessible to dressing, a drop of cod-liver oil can be instilled. This relieves discomfort. An ear-cap of saturated gauze should be applied to keep the dressings in position. The response to this careful dressing is dramatic, and all crusting disappears in 24 to 48 hours. Subsequently the liniment can be simply smeared on without any dressing to cover it.

After discharge the patient is advised to wash or shave over a protective layer of lanette wax base or calamine liniment for a few days.

There still remain a few points on technique to be mentioned. First, the use of jaconet as a covering dressing. By retaining moisture, this economizes labour and material. Twice-daily treatment has sufficed, and fewer layers of gauze are required. Hot weather and the presence of acute inflammation are contraindications to the use of jaconet. It is essential at all times that the dressings remain moist; otherwise, by adhering, they may cause damage.

Pitfalls leading to failure arise from insufficient padding of the natural folds - e.g., fold of chin, angles of mouth, nasolabial folds, and bridge of the nose-and from bandaging that is not firm enough to keep the padding in position. If the angles of the mouth are involved it is an advantage to apply to the upper lip a dressing which is overlapped at the angles of the mouth by the firm lower-jaw bandage. The naso-labial folds should be treated in the same way as the folds of the ear-with a tape dressing. Bandaging must be firm, and to achieve this two half-masks, consisting of goggles and a fourtailed bandage for the chin, are preferable to one face-mask, which would tend to sag in places. The eyebrows also need particular care, and a specially thick firm dressing to ensure the rapid removal of all crusts. Liq. tinctor. applied twice daily to the eyelids will deal effectively with any lesions too close to the eye for bandaging.

The main points in the management of the case have now been dealt with. There is, however, one other item of supreme importance-that of not lightly changing treatment. One change is apt to lead to numberless others, until the medical officer and skin are both bewildered-the skin finally refusing to tolerate treatment. 


\section{Comparison with Sulphonamide Treatment}

The method just described was elaborated by us towards the end of the period during which the local treatment of pyodermia with sulphonamides was fashionable, and we cannot dismiss the subject without further reference to that popular form of treatment. In a series of 643 cases, comprising 364 of impetigo and 279 of infective dermatitis, we recorded, with reference to the local treatment received before admission, the length of stay in hospital before the patient was fit for duty or for discharge into civilian life.

Of the cases of impetigo which had a general average stay in hospital of 14.5 days, 84 had been treated with sulphonamides for an average of 22 days ; of the rest-some of which we ourselves treated with sulphonamides, some with calamine liniment, and some with other remedies-the figures were 11.4, 10.8 , and 12.6 respectively. Of the cases of infective dermatitis, 63 which had been treated elsewhere with sulphonamides took 28.1 days on the average to get well, as compared with $\mathbf{1 8 . 6}$ days for the rest. It will be seen from these figures that the average duration of stay in hospital of the cases we ourselves treated with sulphonamide is scarcely longer than that of the cases treated with calamine liniment. Selection played a part in this. Mild cases for which expenditure of large quantities of liniment and dressing appeared extravagant, and cases of the types considered especially amenable, were treated with sulphonamides, while all the more severe ones had calamine liniment. Nevertheless, we feel it necessary to set out once more in tabular form the advantages and disadvantages of the two methods :

Local Sulphonamides

1. Ease of application (as thick smears, twice daily)

2. Economy-no dressings required

3. Aggravation of local condition encountered not infrequently

4. Risk of delaying response to subsequent treatment

5. Experience required in choosing the type of case likely to respond straightforwardly to treatment

6. Limited safe period of use, 5-7 days; beyond that, increasing risk of sensitization

7. Risk of later serious after-affects (1) sensitization to light; (2)
sensitization to sulphonamides

\section{Summary}

Antiseptics are not necessary in the successful treatment of impetigo. The use of any of them-mercurials, dyes, sulphonamides, etc. - entails the risk of disastrous aggravation of the disease. Unsuccessful treatment with sulphonamides, quite apart from obvious sensitization phenomena, makes subsequent cure more difficult. A method of treatment with a simple calamine liniment is described and recommended for use in severe cases of all kinds of impetigo and infective dermatitis.

We are indebted to Lieut.-Col. Wilson for his interest and the suggestion of extending the application of calamine liniment to the external meatus in the presence of otitis externa; to Maior Twiston Davies, command dermatological specialist, for his untiring help in preparing this paper; and to the nursing staff, especially Sister Joyce, who suggested the ruthless shaving.

The Malta Health Report for 1943 appears over the signature of Dr. A. V. Bernard, Chief Medical Officer. During the period under review there was a rapid and substantial improvement in the public health of Malta and Gozo. Although overcrowding was worse than ever and environmental sanitation far from perfect, the special attention paid to maternal and infant welfare work, the small incidence of infectious disease, and the use of sulphonamide drugs in treatment of respiratory and intestinal infections all helped in reduction of the death rate. Antidiphtheria inoculation brought down the mortality from this disease to the lowest for nineteen years. The slaughtering of many goats during the siege and pasteurization of milk resulted in a lower incidence of undulant fever than ever before. Only one case of indigenous malaria (benign tertian) was observed. War casualties were 5.7 per 1,000 of the population. An explosive outbreak of typhoid fever followed percolation from a bomb-damaged sewer into one of the main water reservoirs. Subsequently an Order was issued for compulsory inoculation. This is the last of a series of reports which deal with actual war conditions in Malta.

\section{Medical Memoranda}

\section{Expulsion of Ectopic Foetus per Rectum}

It is a rarity for a foetus to be expelled per rectum, and for this reason this case is being reported.

\section{CASE Report}

The patient, aged 28, came to the hospital complaining of acute abdominal pain, abdominal swelling, and obstinate constipation. Just before admission she had a severe uterine haemorrhage after a period of three months' amenorrhoea. With this bleeding there were vomiting and inguinal pain. Previously menstruation, which started in her fourteenth year, had always been regular. In this year she married and for ten years she remained sterile. Her year she married and for ten
husband died and she remarried.

On admission the temperature was normal and the pulse 90. The patient was pale. Abdominal examination revealed a hard mass rising out of the pelvis and reaching half-way to the umbilicus. Per vaginam the uterus was found to be anteflexed, and the fundus was felt one finger above the pubis. The portio vaginalis was was felt one finger above the pubis. The portio vaginalis was conical and the cervix closed. The posterior fornix was distended Douglas. Posterior colpotomy was performed, but no discharge followed this. Pelvic haematocele was diagnosed and the patient treated conservatively. For six days the temperature remained at $39^{\circ} \mathrm{C}$. As there was no improvement operation was advised, but the patient refused and left hospital.

A fortnight later she returned complaining of rectal haemorrhage and stating that she had passed a foetus per rectum two days

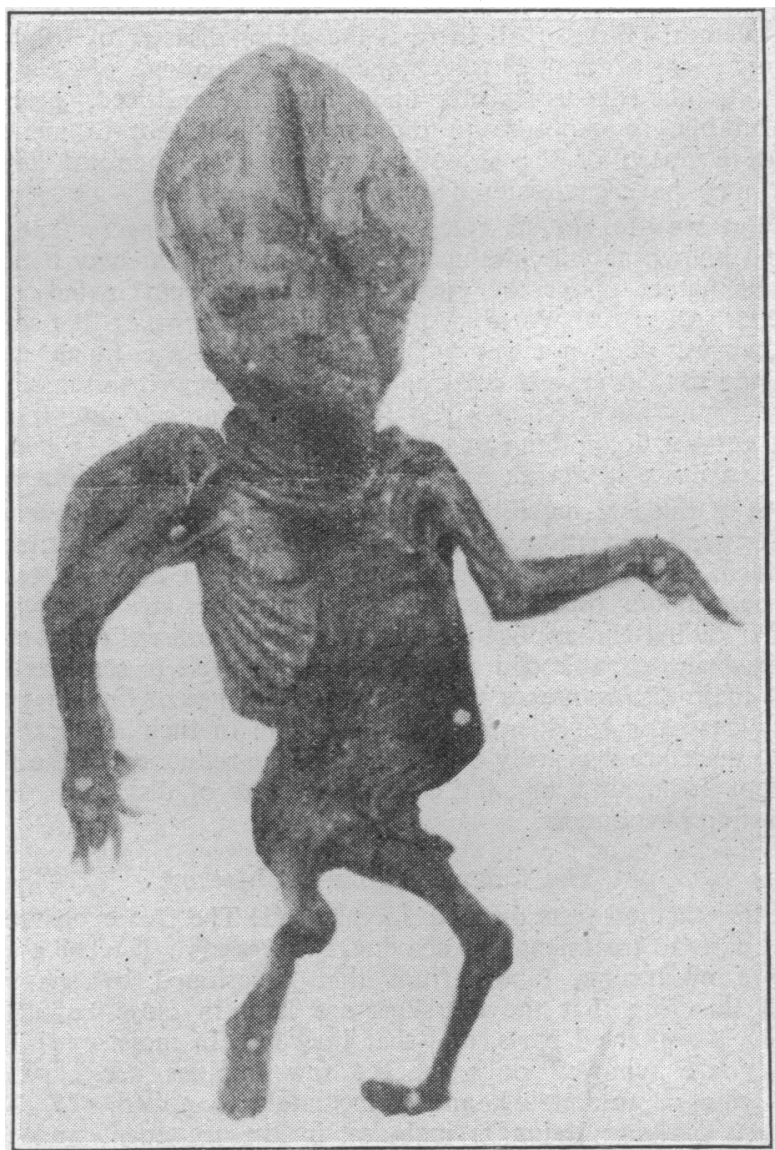

previously. The foetus (see photograph) was $9 \mathrm{~cm}$. in length and mummified. On rectal examination a fistula could be felt, $3 \mathrm{~cm}$. in diameter, on the anterior wall about 7 to $8 \mathrm{~cm}$. from the anus. Through the fistula the finger entered the large sac of the haematocele. There was a blood-stained blackish discharge. For 22 days the temperature varied between 37 and $38^{\circ} \mathrm{C}$. At the end of this time she was examined again. The uterus was deviated to the left, but the left fornix was normal. On the right side there was a mass, the size of a fist, attached to the pelvic wall. The patient made an uninterrupted recovery.

From the literature available there has been a similar type of case reported (Binagh and Bardi, 1929), but the whole of the foetus was not expelled per rectum.

Smyrna Hospital. 